\title{
A New Coordinated Control Method on the Intersection of Traffic Region
}

\author{
Minghui Ma, Qingfang Yang, Shidong Liang, and Yashi Wang \\ College of Transportation, Jilin University, Changchun 130022, China \\ Correspondence should be addressed to Shidong Liang; liangsd14@mails.jlu.edu.cn
}

Received 23 November 2015; Revised 20 March 2016; Accepted 22 March 2016

Academic Editor: Alicia Cordero

Copyright (C) 2016 Minghui Ma et al. This is an open access article distributed under the Creative Commons Attribution License, which permits unrestricted use, distribution, and reproduction in any medium, provided the original work is properly cited.

\begin{abstract}
Intersection regions widely exist in road networks as crucial connections, and the congestion effects on traffic in the networks cannot be ignored. In general, a conventional intersection region includes a merging region and more than two ramps, whose congestion brings a serious negative effect on traffic service quality and satisfaction of drivers. Specifically, intersection region congestion is caused by congestion in merging regions. However, current control methods regarding coordination of the variable speed limits and ramp metering in order to solve merging region congestion are insufficient to handle congestion occurring in the intersection region. Therefore, a new method of coordinated control is proposed in this paper based on mainline control using the variable speed limits and coordinated ramp metering. The coordinated control method is formulated considering the degree of crowdedness indexes and implemented based on the queue length, with the goal to relieve congestion in conventional intersection regions caused by merging region congestion. The coordinated control method is further evaluated based on an actual traffic network. The results show that the proposed method is effective in terms of improving traffic service quality and relieving intersection region congestion.
\end{abstract}

\section{Introduction}

Congestion in road networks becomes more noticeable in modern societies, bringing negative effects on sustainable development of the Intelligent Transportation System (ITS), such as traffic service quality, fuel consumption, and traffic costs and benefits. In general, traffic congestion can be solved or relieved by constructing a new road or developing road facilities, but these methods are costly, labor-intensive, and time-consuming. Therefore, it is necessary to focus on better utilization of the existing road infrastructure using scientific and reasonable traffic control methods to mitigate congestion and improve service quality. In the past decades, numerous control methods and strategies were developed and implemented to relieve congestion in networks.

The variable speed limits (VSL) control method, as a new traffic control measure, is widely applied on freeway mainlines to solve the traffic congestion problem [1-3]. The reliability and practicability of the VSL is validated in previous studies using numerical and dynamic simulations of power systems [4-6]. The VSL can be defined as a dynamic control method using dynamic changes in reasonable VSL values to improve current traffic conditions. The VSL values, as the core of the VSL control method, are calculated in diverse traffic conditions involving traffic flows, traffic density, and so forth. It is recognized that VSL control is an effective method of improving the security and efficiency of freeway networks [7-15]. However, when heavy traffic congestion occurs in a regional network, especially on the intersection of traffic regions, VSL control becomes inefficient for solving the traffic problem. Therefore, to overcome these limitations of the method and solve the congestion problem in complicated networks, coordinated control methods involving VSL control and ramp metering have gradually drawn attention by scholars.

Coordinated methods involving VSL control and ramp metering were investigated in [16-20], where their validity in solving the congestion problem in independent merging regions has been proved. It is necessary to state that an independent merging region is defined as slight congestion presented in the merging region. Although a lot of transportation studies were dedicated to this topic, such as $[21,22]$, 


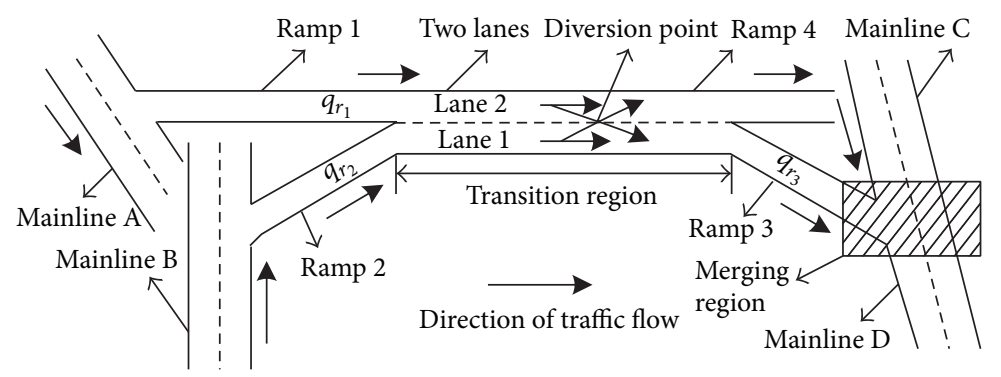

FIGURE 1: Studied network.

there are some difficulties in applying these proposed methods based on some complicated models, such as calibration of parameters and model training. At the same time, in previous coordinated control approaches, such as [23-25], simulation results were not satisfactory. Furthermore, most of the coordinated control methods combining VSL control with ramp metering were proposed to improve congestion in independent merging regions of freeway networks, such as [26, 27]. However, when heavy traffic congestion exists in a dependent merging region where the in-ramp connects to another major traffic road, current coordinated control methods involving VSL control and ramp metering are invalid. Congestion in a dependent merging region existing widely in freeway networks has some negative effects on the on-ramp upstream of other roads, such as lower efficiency of traffic operation and shortage of services supply. Therefore, to propose a simple and effective control method to solve this congestion problem is necessary and important.

In this paper, a new optimal coordinated control method involving VSL control and coordinated ramp metering is proposed to solve the congestion problem of conventional intersection regions. Specifically, the queue length in each link of the studied network is considered as a threshold parameter to meet the requirement of the coordinated control strategy. In addition, because traffic conditions can be described well by traffic density fluctuations, the traffic density parameter is chosen as the primary parameter to construct the evaluation index measuring the degree of crowdedness. Further, correction of errors is considered to adjust the process of coordinated control. The contributions of this paper can be summarized as follows.

(i) A universal congestion problem of conventional intersections of road networks is considered, and traffic operation characteristics of studied networks are analyzed and studied.

(ii) A novel coordinated control strategy is proposed, and the queue length in each link of the studied network is considered as a threshold parameter.

(iii) The degree of crowdedness evaluation index is constructed based on the density parameter, which is the core value of the coordinated control method proposed in this paper. The index is used to adjust the priority of the outflow of each link in the studied network. The greater the degree of crowdedness, the more the right of way that should be given to the link.
This paper is organized as follows. Section 2 introduces the research freeway region and describes the traffic congestion problem. In Section 3, a novel coordinated control method is proposed. Section 4 presents a real traffic network and several illustrative results. Finally, in Section 5, conclusions and some topics for further research are stated.

\section{Traffic Problem of Application Network}

In this paper, the intersection region of a road network is considered; see Figure 1. The studied network includes a merging region and several ramps, so that the network is quite complicated compared to a regular merging region and has inevitable congestion during peak hours. In order to present a reasonable and suitable control method and strategy, it is crucial to give a detailed description and explanation of traffic operation in the intersection region.

As shown in Figure 1, there are three mainlines and three ramps in the studied network. Specifically, the three ramps include one on-ramp, 3, and two off-ramps, ramps 1 and 2. When the traffic demand becomes higher than the capacity of the merging region, congestion occurs in the intersection region. The process of congestion based on the diffusion of congestion can be divided into three parts: congestion of the merging region, congestion of ramp 3, and congestion of the transition region. The process of congestion formed during the peak period in the intersection region is discussed below.

Traffic congestion first takes place in the merging region of the studied network, depicted in Figure 2(a). If the arriving flow coming from both mainline $\mathrm{C}$ and ramp 3 is higher than the merging region capacity $q_{\text {mer,cap }}$, the traffic problem in the merging region appears. Then, there is a queue that forms in mainline $\mathrm{C}$ and ramp 3. While the traffic demand in the merging region continues to increase, overcrowding at ramp 3 starts causing negative influence on transition region traffic operation.

Figure 2(b) shows a transition region upstream of the merging region. When the queue length exceeds the length of ramp 3 during the peak period, congestion appears in the transition region. At the same time, the right of way of ramp 3 vehicles flowing into the merging region is restricted by common rules. The traffic congestion in the transition region appears. Vehicles in the transition region queue that come from ramp 1 are denoted as $q_{r_{1}}$ and from ramp 2 as $q_{r_{2}}$. Specifically, in the transition region, the partial outflow of ramps 1 and 2 finishes the interleave behavior. 


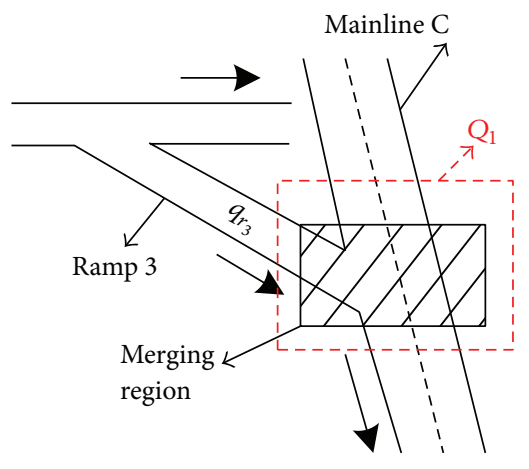

(a) The congestion region $Q_{1}$

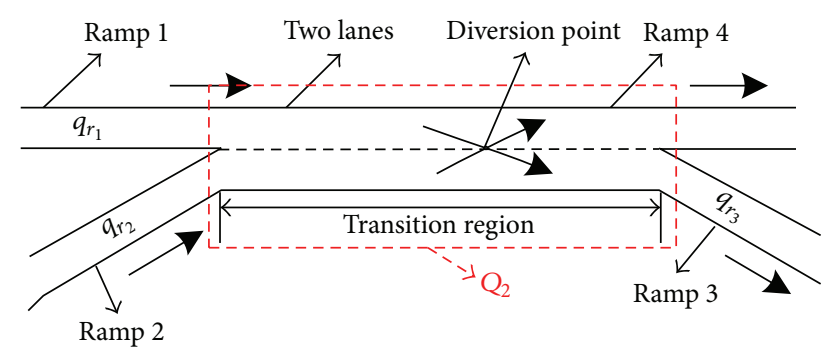

(b) The congestion region $Q_{2}$

Figure 2: Traffic problem decomposition diagrams.

There is a diversion point existing in the transition region dynamically which is defined as a decision point whether the vehicles flowing into the on-ramp 3 , which can be seen in Figures 1 and 2(b). In the beginning, the queue phenomenon only exists in lane 1 of the transition region. With an increase in the queue length of lane 1, the diversion point moves up continuously along the transition section. Once the diversion point arrives at the position close to ramps 1 and 2, congestion occurs in ramps 1 and 2 . However, if the inflow of ramps 1 and 2 continues to increase, the spillback queue of ramps 1 and 2 has negative influence on mainline A and mainline B traffic operation.

Congestion in transition regions is universal in road networks with the increase in car ownership. Using the above analysis, we can see that the merging region is presented as the origin of congestion in the transition region. In this studied network, merging region congestion not only affects merging region traffic operation, but also influences traffic conditions of the adjoining regions and the whole regional network. Therefore, a reasonable and effective traffic control method is crucial to relieving congestion in transition regions of road networks.

\section{Formulation of the Coordinated Control Method}

The studied network is represented by a directed graph where the links of the graph represent stretches, as shown in Figure 1. According to the discussion of traffic operation in Section 2, traffic congestion in the studied network is a complicated problem. Therefore, in order to enhance traffic service quality, a novel coordinated control method including a variable speed limit and coordinated ramp metering is proposed. Specially, the VSL control region is placed at a particular place of the mainline $\mathrm{C}$, and coordinated ramp metering points are located in ramp 1, ramp 2, and ramp 3, respectively. The coordinated control method and strategy are presented as follows.

Due to different situations created by different processes of congestion, the coordinated control strategy also involves three parts based on the queue length in the mainline $\mathrm{C}$, ramp, and transition region. In the coordinated control method, an algorithm for computing the queue length is required, so we use the queue length calculation method proposed by [28], where the accuracy of the queue algorithm is discussed in their work. According to the queue length in each section of the studied network, the control strategy is presented, and the coordinated control method is formulated.

With the continuity of ramp 3 queue into the transition region, the total queue length is defined as $w_{\text {Tran }}(k)$ (veh). According to different queue lengths $w_{\text {Tran }}(k)$, the coordination control method includes three cases.

Scenario 1. The queue length $w_{\operatorname{Tran}}(k)$ is less than the capacity of the on-ramp $r_{3} N_{r 3, \mathrm{C}}$, which means $w_{\text {Tran }}(k)<(1-\alpha) N_{r 3, \mathrm{C}}$ (traditional $\alpha$ is $1 / 3$ ).

In this scenario, the traffic operation of the on-ramp $r_{3}$ has little influence on the transition region traffic flow. The traffic congestion in the merging region, as shown in Figure 2(a), should be, first, solved by the coordinated control method that involves VSL control and ramp metering, which is regular traffic flow. The previous studies almost do not take account of the right of way of on-ramp $r_{3}$ vehicles flowing into the merging region. However, it is not fair to restrict the right of way to the merging region of ramp 3 vehicles when serious traffic congestion exists in ramp 3.

Therefore, the releasing rate index is proposed to adjust this problem. The releasing rate can be obtained using the degree of crowdedness in on-ramp $r_{3}$ and upstream mainline $\mathrm{C}$ of the merging region. In Scenario 1, the degree of crowdedness indexes of mainline $\mathrm{C}$ and on-ramp $r_{3}$ are defined as $\xi_{\mathrm{mC}}(k)$ and $\xi_{\mathrm{r} 3}(k)$, respectively, given by the following equations:

$$
\begin{gathered}
\xi_{r 3}(k)=\frac{\rho_{r 3}(k) \rho_{\mathrm{mC}, J}}{\rho_{r 3}(k) \rho_{\mathrm{mC}, J}+\rho_{\mathrm{mC}}(k) \rho_{r 3, J}}, \\
\xi_{\mathrm{mC}}(k)=\frac{\rho_{\mathrm{mC}}(k) \rho_{r 3, J}}{\rho_{r 3}(k) \rho_{\mathrm{mC}, J}+\rho_{\mathrm{mC}}(k) \rho_{r 3, J}},
\end{gathered}
$$

where $\xi_{r 3}(k)$ and $\xi_{\mathrm{mC}}(k)$ are the congestion indexes of ramp $r_{3}$ and mainline $C$ during the period $k$, respectively; $\rho_{r 3}(k)$ and $\rho_{\mathrm{mC}}(k)$ are the density of the on-ramp $r_{3}$ and mainline C, respectively; and $\rho_{r 3, J}$ and $\rho_{\mathrm{mC}, J}$ are the jam density of 
the on-ramp $r_{3}$ and mainline $\mathrm{C}$, respectively. Specifically, $\rho_{r 3}(k)$ and $\rho_{\mathrm{mC}}(k)$ can be calculated by the following equations:

$$
\begin{gathered}
\rho_{r 3}(k)=\frac{N_{r 3}(k)}{L_{r 3}}, \\
\rho_{\mathrm{mC}}(k)=\frac{N_{\mathrm{mC}}(k)}{\lambda_{\mathrm{mC}} L_{\mathrm{mC}}},
\end{gathered}
$$

where $N_{r 3}(k)$ and $N_{\mathrm{mC}}(k)$ are the numbers of vehicles in the $\operatorname{ramp} r_{3}$ and mainline $C$ during the period $k$, respectively; and $L_{r 3}$ and $L_{\mathrm{mC}}$ are the length of the on-ramp $r_{3}$ and mainline C, respectively. Specifically, the $N_{r 3}(k)$ and $N_{\mathrm{mC}}(k)$ can be given by

$$
\begin{aligned}
N_{r 3}(k) & =q_{\mathrm{in}, r 3}(k) T+w_{r 3}(k), \\
N_{\mathrm{mC}}(k) & =T \sum_{i=1}^{k}\left(q_{\mathrm{in}, \mathrm{mC}}(i)-q_{\mathrm{out}, \mathrm{mer}}(i)+q_{\mathrm{out}, r 3}(i)\right),
\end{aligned}
$$

where $q_{\text {in, } r 3}(k)$ is the inflow of on-ramp $r_{3} ; w_{r 3}(k)$ is the queue length of on-ramp $r_{3} ; T$ is the time moment; $q_{\text {out }, r 3}(i)$ is the outflow of on-ramp $r_{3}$; and $q_{\text {out,mer }}(i)$ is the outflow of the merging region, $0 \leq i \leq k$. The on-ramp $r_{3}$ inflow includes two parts: one part comes from ramp $r_{1}$, and the other part is from ramp $r_{2}$, which can be presented as follows:

$$
\begin{aligned}
q_{\text {in }, r 3}(k)= & q_{\text {out }, r 1}\left(k-1-\frac{L_{\operatorname{Tran}}}{v_{\operatorname{Tran}, f} T}\right) \delta_{r 1, \operatorname{Tran} 1} \\
& +q_{\text {out }, r 2}\left(k-1-\frac{L_{\operatorname{Tran}}}{v_{\operatorname{Tran}, f} T}\right) \delta_{r 2, \operatorname{Tran} 1},
\end{aligned}
$$

where $q_{\text {out }, r 1}\left(k-1-L_{\operatorname{Tran}} / v_{\operatorname{Tran}, f} T\right)$ and $q_{\text {out }, r 2}(k-1-$ $\left.L_{\text {Tran }} / \nu_{\text {Tran, } f} T\right)$ are the traffic flow transferred to on-ramp $r_{3}$ from ramp $r_{1}$ and ramp $r_{2}$, respectively, and $\delta_{r 1, \operatorname{Tran} 1}$ and $\delta_{r 2, \operatorname{Tran} 1}$ are the shunt rates of the traffic flows leaving ramp $r_{1}$ and ramp $r_{2}$ to ramp $r_{3}$, respectively. Specifically, $1+L_{\operatorname{Tran}} / v_{\operatorname{Tran}, f} T$ is a time delay; $L_{\operatorname{Tran}}$ is the length of the transition region; and $v_{\operatorname{Tran}, f}$ is the free-flow speed in the transition region.

Considering the degree of crowdedness index proposed above, the number of discharging vehicles from mainline $\mathrm{C}$ and on-ramp $r_{3}$ into the merging area can be determined by (5) and (6), respectively, which can be expressed by

$$
\begin{gathered}
X_{\mathrm{out}, \mathrm{r} 3}(k)=T \frac{q_{\mathrm{mer}, \mathrm{cap}} \rho_{r 3}(k) \rho_{\mathrm{mC}, J}}{\rho_{r 3}(k) \rho_{\mathrm{mC}, J}+\rho_{\mathrm{mC}}(k) \rho_{r 3, J}}, \\
X_{\mathrm{out}, \mathrm{mC}}(k)=T \frac{\mu q_{\mathrm{mer}, \mathrm{cap}} \rho_{\mathrm{mC}}(k) \rho_{r 3, J}}{\rho_{r 3}(k) \rho_{\mathrm{mC}, J}+\rho_{\mathrm{mC}}(k) \rho_{r 3, J}},
\end{gathered}
$$

where $X_{\text {out }, r 3}(k)$ and $X_{\text {out,mC }}(k)$ are the number of vehicles flowing out from on-ramp $r_{3}$ and mainline $\mathrm{C}$, respectively, and $\mu$ is the model parameter determined by the traffic demand. A conventional constraint that the total outflow of on-ramp $r_{3}$ and mainline $\mathrm{C}$ should be limited by the capacity of the merging region can be presented as follows:

$$
X_{\text {out }, r 3}(k)+X_{\text {out }, \mathrm{mC}}(k) \leq q_{\text {mer,cap }} T .
$$

According to (7), the outflows of on-ramp $r_{3}$ and mainline $\mathrm{C}$ can be obtained. Furthermore, the variable speed limit values and ramp metering rates can be obtained from

$$
\begin{aligned}
X_{\mathrm{out}, r 3}(k) & =\gamma_{\mathrm{r} 3}(k) q_{\mathrm{out}, r 3}(k) T, \\
X_{\mathrm{out}, \mathrm{mC}}(k) & =\lambda_{\mathrm{mC}} \rho_{\mathrm{mC}}(k) v_{\mathrm{vsl}}(k) T,
\end{aligned}
$$

where $\gamma_{r 3}(k)$ is the on-ramp $r_{3}$ metering rate; $\lambda_{\mathrm{mC}}$ is the number of mainline C lanes; $v_{\mathrm{vsl}}(k)$ is the VSL value. According to (8) and (9), the VSL values $v_{\mathrm{vsl}}(k)$ and the on-ramp $r_{3}$ releasing rate $\gamma_{r 3}(k)$ during the period $k$ can be obtained.

Scenario 2. The queue length $w_{\operatorname{Tran}}(k)$ is larger than the capacity of on-ramp $r_{3} N_{r 3, C}$, while $w_{\text {Tran }}(k)$ is lower than the demand of both transition region lane 1 and on-ramp $r_{3}$. This means that $(1-\alpha) N_{r 3, \mathrm{C}} \leq w_{\text {Tran }}(k)<N_{\text {Tran,C }}-\Delta s_{\text {Tran }}+N_{r 3, \mathrm{C}}$, where $N_{\text {Tran,C }}$ is the capacity of transition region lane 1 and $\Delta s_{\text {Tran }}$ is a buffer parameter.

In Scenario 2, the on-ramp $r_{3}$ queuing vehicles reach the transition region and continue to grow along lane 1 of the transition region. That is, the heavy inflow of onramp $r_{3}$ causes congestion in Scenario 2 and may further result in serious traffic problems in the studied network. With the queue length increasing, the diversion point moves backward. In order to stop spoilage of the traffic network, the coordinated control method in Scenario 1 is applied first. However, Scenario 2 is different from Scenario 1. Therefore, the conventional operation rule has to be modified. It is crucial that more right of way must be given to the on-ramp $r_{3}$ outflow in Scenario 2. Specifically, the outflow of on-ramp $r_{3}$ represents real freedom $\left(\gamma_{r 3}(k)=1\right)$. Therefore, the mainline C outflow from upstream can be calculated as follows:

$$
q_{\text {out }, \mathrm{mC}}(k)=q_{\text {mer,cap }}-q_{r 3, \text { cap }}(k),
$$

where $q_{\text {out,mC }}(k)$ is the outflow of mainline C during the period $k$.

According to (10), the mainline $\mathrm{C}$ outflow equals the difference between the merging region capacity and the maximum outflow of on-ramp $r_{3}$. Because the traffic flow of mainline $\mathrm{C}$ is controlled by the VSL, the mainline $\mathrm{C}$ outflow can also be obtained by the following equation:

$$
q_{\text {out }, \mathrm{mC}}(k)=\lambda_{\mathrm{mC}} \rho_{\mathrm{mC}}(k-\varphi) v_{\mathrm{vsl}}(k-\varphi),
$$

where the time step $k-\varphi$ equals $k-1-L_{\text {vsl-mer }} / v_{\text {vsl-mer, } f} T$; specifically, $1+L_{\mathrm{vsl}-\mathrm{mer}} / v_{\mathrm{vsl}-\mathrm{mer}, f} T$ is a time delay, and $L_{\mathrm{vsl}-\mathrm{mer}}$ and $v_{\mathrm{vsl}-\mathrm{mer}, f}$ are the VSL control region length and the freeflow speed of the VSL control region, respectively.

Scenario 3. The queue length $w_{\operatorname{Tran}}(k)$ is larger than the demand of transition region lane 1 and on-ramp $r_{3}$, while $w_{\text {Tran }}(k)$ is lower than the capacity of transition region lane 1 and on-ramp $r_{3}$, which means $N_{\text {Tran,C }}-\Delta s_{\text {Tran }}+N_{r 3, \mathrm{C}}<$ $w_{\text {Tran }}(k) \leq N_{r 3, \mathrm{C}}+N_{\text {Tran }, \mathrm{C}}$.

Heavy congestion in the transition region may influence normal operation of vehicles in ramp $r_{1}$ and $\operatorname{ramp} r_{2}$. The onramp $r_{3}$ outflow tries to keep full-load operation, and the VSL 
values can be calculated using (9). In Scenario 3, the outflows of ramp $r_{1}$ and ramp $r_{2}$ have to be controlled to prevent the long queue in the transition region from reaching ramp $r_{1}$ and ramp $r_{2}$.

To ensure high efficiency of transition region lane 2 and solve the congestion problem in transition region lane 1, the degree of crowdedness indexes based on traffic density are introduced. In this scenario, the control strategy is that the greater the degree of crowdedness index, the lower the right of way. The degree of crowdedness index reflects the number of vehicles travelling to on-ramp $r_{3}$ eventually. Ramp $r_{1}$ and ramp $r_{2}$ crowdedness indexes can be calculated as follows:

$$
\begin{aligned}
& \psi_{r 1}(k)=\frac{\rho_{r 1}(k) \rho_{r 2, J} \delta_{r 1, \operatorname{Tran} 1}}{\rho_{r 1}(k) \rho_{r 2, J} \delta_{r 1, \operatorname{Tran} 1}+\rho_{r 2}(k) \rho_{r 1, J} \delta_{r 2, \operatorname{Tran} 1}}, \\
& \psi_{r 2}(k)=\frac{\rho_{r 2}(k) \rho_{r 1, J} \delta_{r 2, \operatorname{Tran} 1}}{\rho_{r 1}(k) \rho_{r 2, J} \delta_{r 1, \operatorname{Tran} 1}+\rho_{r 2}(k) \rho_{r 1, J} \delta_{r 2, \operatorname{Tran} 1}},
\end{aligned}
$$

where $\psi_{r 1}(k)$ and $\psi_{r 2}(k)$ are the degree of crowdedness indexes of ramp $r_{1}$ and $\operatorname{ramp} r_{2}$, respectively; $\rho_{r 1}(k)$ and $\rho_{r 2}(k)$ are the density of ramp $r_{1}$ and ramp $r_{2}$, respectively; $\rho_{r 1, J}$ and $\rho_{r 2, J}$ are the jam density of ramp $r_{1}$ and ramp $r_{2}$, respectively. Specifically, $\rho_{r 1}(k)$ and $\rho_{r 2}(k)$ can be obtained by

$$
\begin{aligned}
\rho_{r 1}(k) & =\frac{N_{r 1}(k)}{L_{r 1}}, \\
\rho_{r 2}(k) & =\frac{N_{r 2}(k)}{L_{r 2}}, \\
N_{r 1}(k) & =\sum_{i=1}^{k}\left(q_{\text {in }, r 1}(i)-q_{\text {out }, r 1}(i)\right), \\
N_{r 2}(k) & =\sum_{i=1}^{k}\left(q_{\text {in }, r 2}(i)-q_{\text {out }, r 2}(i)\right),
\end{aligned}
$$

where $N_{r 1}(k)$ and $N_{r 2}(k)$ are the number of vehicles on ramps $r_{1}$ and $r_{2}$, respectively; $L_{r 1}$ and $L_{r 2}$ are the lengths of ramp $r_{1}$ and ramp $r_{2}$, respectively; $q_{\mathrm{in}, r 1}(i)$ and $q_{\mathrm{out}, r 1}(i)$ are the ramp $r_{1}$ traffic inflow and outflow, respectively; and $q_{\mathrm{in}, r_{2}}(i)$ and $q_{\text {out }, r 2}(i)$ are the ramp $r_{2}$ traffic inflow and outflow, respectively.

The number of releasing vehicles of ramp $r_{1}$ and $\operatorname{ramp} r_{2}$ can be given by

$$
\begin{aligned}
& X_{r 1}(k)=\left(N_{r 3, \mathrm{C}}+N_{\text {Tran, } \mathrm{C}}-w_{\text {Tran }}(k)\right) \psi_{r 1}(k) \kappa, \\
& X_{r 2}(k)=\left(N_{r 3, \mathrm{C}}+N_{\text {Tran, } \mathrm{C}}-w_{\text {Tran }}(k)\right) \psi_{r 2}(k),
\end{aligned}
$$

where $\kappa$ is a model parameter determined by the traffic demand.

The number of releasing vehicles from ramp $r_{1}$ and ramp $r_{2}$, in Scenario 3, can be obtained from (12) to (14). Since ramp metering is adopted to control ramp $r_{1}$ and ramp $r_{2}$ outflows, the outflows of ramp $r_{1}$ and ramp $r_{2}$ can also be obtained from

$$
\begin{aligned}
& X_{\text {out }, r 1}(k)=\gamma_{r 1}(k) q_{\text {out }, r 1}(k) T, \\
& X_{\text {out }, r 2}(k)=\gamma_{r 2}(k) q_{\text {out }, r 2}(k) T,
\end{aligned}
$$

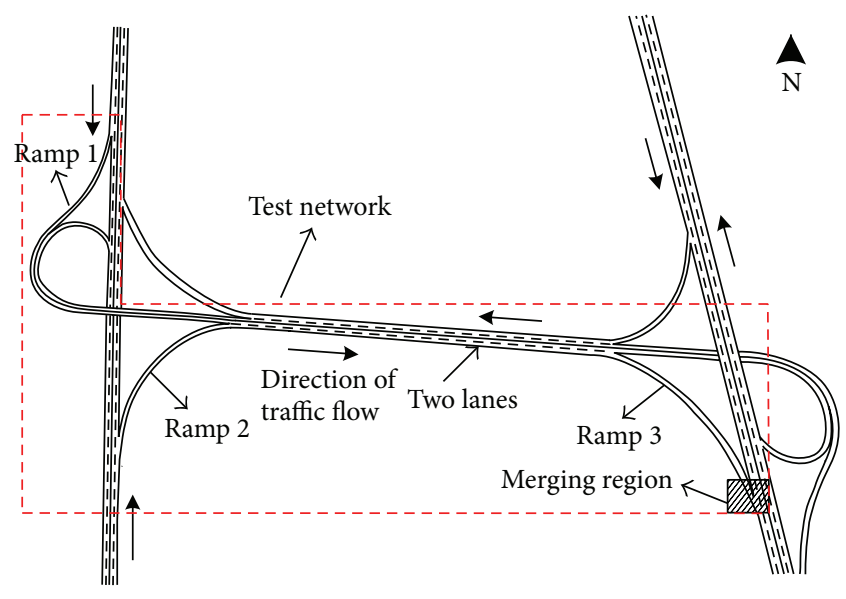

FIGURE 3: Study site of freeway network.

where $\gamma_{r 1}(k)$ and $\gamma_{r 2}(k)$ are the metering rates of ramp $r_{1}$ and ramp $r_{2}$, respectively.

The three scenarios above show the process of traffic state changes. The coordinated control strategies are presented in the above discussion. The ultimate goal of the coordinated control strategy is to ensure normal operation of the traffic network by ensuring the maximum outflow of the merging region and easing the queue in on-ramp $r_{3}$. Since some disturbing factors exist in real situations, the actual outflow from the merging region is lower than the one calculated. Therefore, it is necessary to eliminate or relieve actual disturbances in calculations. The disturbance parameter can be calculated using

$$
e_{\chi}(k)=\widehat{q}_{\text {out }, \chi}(k)-q_{\text {out }, \chi}(k),
$$

where $e_{\chi}(k)$ is the disturbance parameter and $q_{\text {out }, \chi}(k)$ is the outflow from each section in the studied network. Accordingly, $q_{\text {out }, \chi}(k)$ can be described as follows:

$$
q_{\mathrm{out}, \chi}(k)=F+K_{\chi} e_{\chi}(k),
$$

where $F$ is the outflow calculated by former equations and $K_{\chi}$ is an adjusting parameter.

\section{Application Results}

4.1. Experiment Design and Test Network. To demonstrate supposed effectiveness of the proposed control method, two cases involving the no-control and coordinated control methods are applied in the experiment. In particular, the settings of the above scheme are designed as follows: the nocontrol case is the case without any additional traffic control, and the coordinated control case adopts the coordinated control method proposed in this paper.

In order to achieve the purpose of this study, an actual regional network G104 and G3 in China is considered for evaluation of efficiency and suitability of the coordinated control method. There is a static posted speed limit of $100 \mathrm{~km} / \mathrm{h}$. The freeway test-bed is shown in Figure 3. This test freeway network always has recurrent heavy congestion 


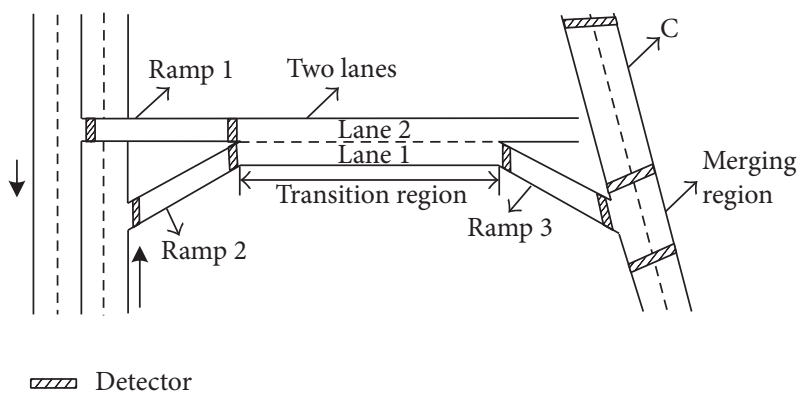

FIGURE 4: Detectors distribution.

during the afternoon peak hours due to high traffic demand. The test network includes three ramps, a transition region, and a merging region.

The data used for calibrating the model was collected from video detectors of the studied network. The density can be determined by analyzing fundamental relations among traffic variables. There are nine traffic loop detectors located in this test freeway network, shown in Figure 4, which is a simple framework diagram. Ramp $r_{1}$ and ramp $r_{2}$ lengths are set to 700 meters and 300 meters, respectively. On-ramp $r_{3}$ length is set to 380 meters, and the transition region is set to 983 meters. The single-lane capacity is $1750 \mathrm{veh} / \mathrm{h}$. The curtail density of a single-lane is $33 \mathrm{veh} / \mathrm{km}$. The VSL region length is 250 meters. The length of the section between the VSL region and merging region is 500 meters, according to [18]. The parameters are set as follows: $\alpha=1 / 3, \Delta s_{\text {Tran }}=588$ meters, and $\kappa=0.75$. The shunt rates $\delta_{r 1, \operatorname{Tran} 1}$ and $\delta_{r 2, \operatorname{Tran} 1}$ are equal to $47 \%$ and $52 \%$, respectively. The free-flow speed of the transition region and mainline are $75 \mathrm{~km} / \mathrm{h}$ and $91 \mathrm{~km} / \mathrm{h}$, respectively.

4.2. Results Analysis. The studied regional network is formulated in a simulation platform. In this section we show the results of simulations under two different conditions: the nocontrol case and the coordinated control case. The evaluation results are presented in what follows.

Figure 5 shows the outflow of the merging region. Figures 6 and 7 are the outflows of ramps 1 and 2 under the two control cases, respectively. Figures 8 and 9 are the travel speeds of ramps 1 and 2 under the two control cases, respectively. The queue length of each segment of the studied network is presented in Figure 10. Figures 11 and 12 show the VSL values and ramp metering releasing rates under the coordinated control method, respectively.

Figure 5 shows the merging region outflow under the no-control and coordinated control cases. As can be seen in Figure 5, under the coordinate control case, the merging region outflow is higher than that under the no-control case. Specifically, during the period from $2700 \mathrm{~s}$ to $3300 \mathrm{~s}$, there is a significant drop in the merging region outflow curve under the no-control case of about $6.7 \%$. In comparison, the merging region outflow under the coordinated control case decreased only slightly by $2.8 \%$. During the period from $3300 \mathrm{~s}$ to $7600 \mathrm{~s}$, the average outflows from the merging region under two control cases are $3176 \mathrm{veh} / \mathrm{h}$ and $3259 \mathrm{veh} / \mathrm{h}$, respectively. Specifically, the average outflow of the merging

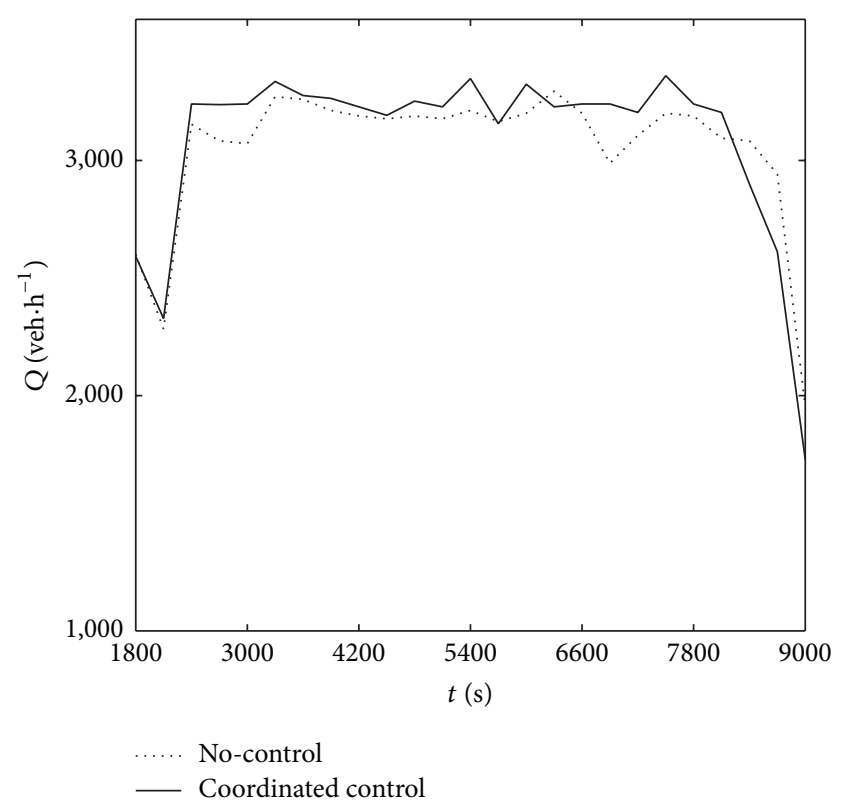

Figure 5: Merging region outflow.

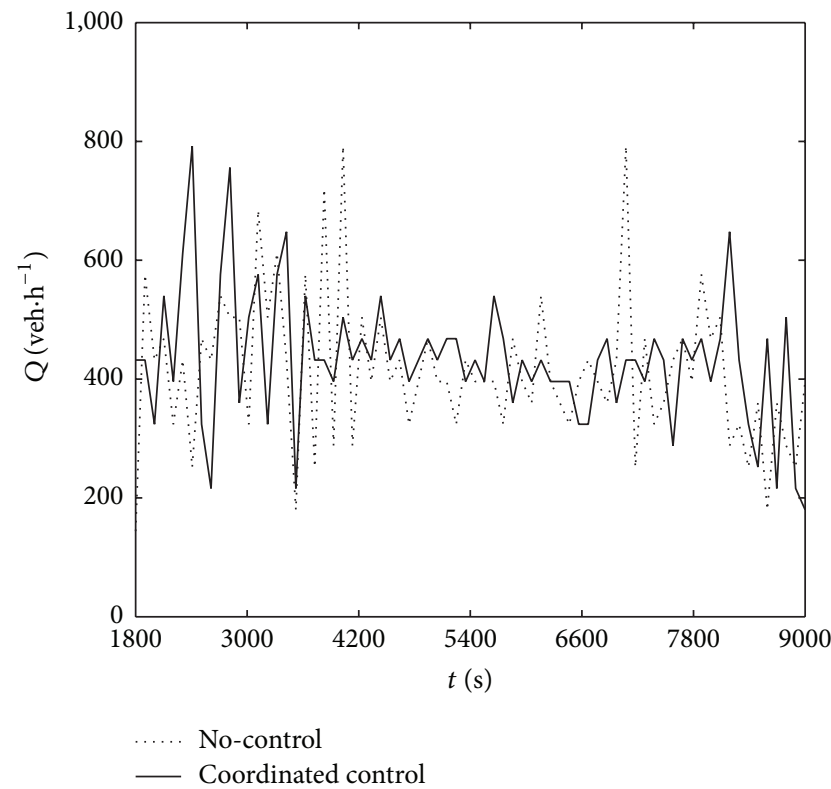

FIgURE 6: Ramp 1 outflow.

region under the coordinated control case represents $2.8 \%$ improvement compared to the no-control case.

Figures 6 and 7 show variations in ramps 1 and 2 outflows, respectively. During the peak period, the traffic flows in ramps 1 and 2 are steady under the coordinated control case compared to the no-control case.

Figure 8 shows the travel speeds of ramp 1. As a whole, the illustrations reveal that the travel speeds of ramp 1 are clearly improved under the coordinated control case compared to the no-control case. In the peak period, especially, the travel speeds of ramp 1 fluctuate within the range of $10.4 \mathrm{~km} / \mathrm{h} \sim 23.8 \mathrm{~km} / \mathrm{h}$ under the no-control case and $19.1 \mathrm{~km} / \mathrm{h} \sim 47.3 \mathrm{~km} / \mathrm{h}$ under the other case, and the travel 


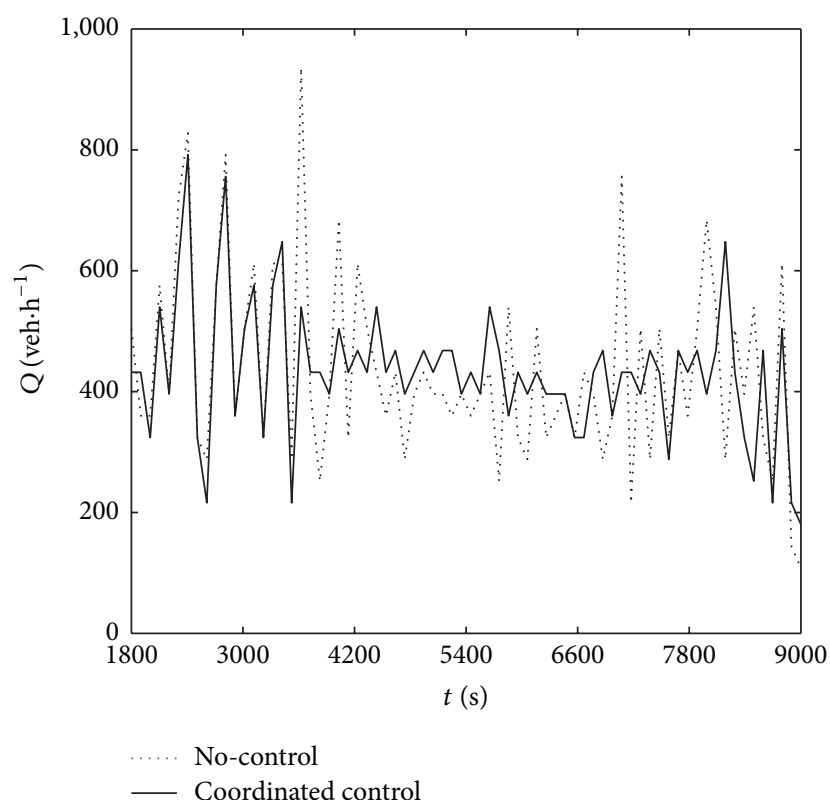

Figure 7: Ramp 2 outflow.

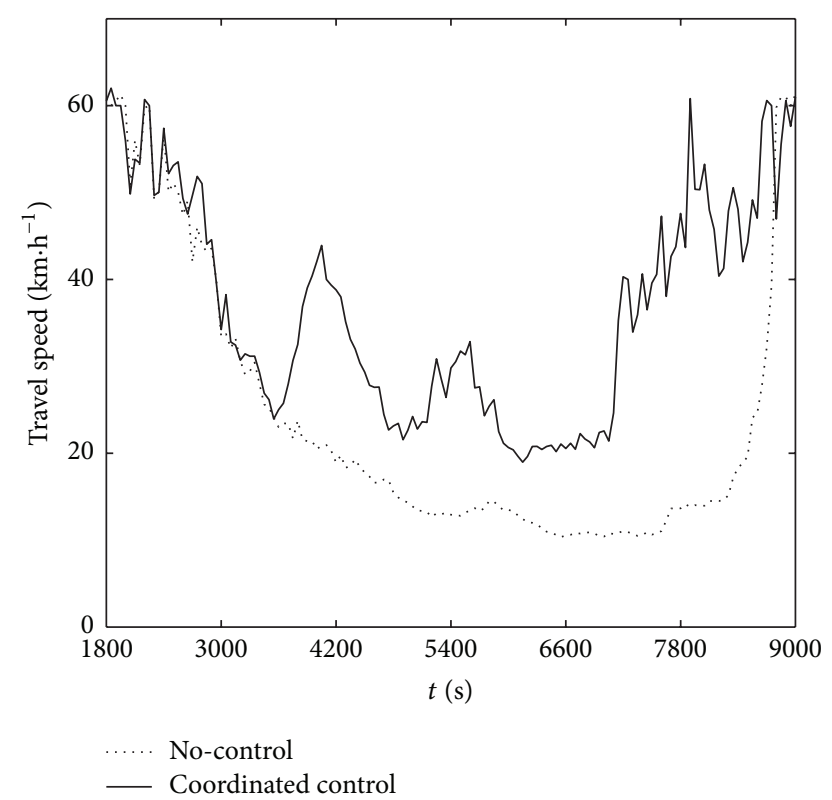

FIGURE 8: Ramp 1 travel speed.

speeds of ramp 1 have a clear downtrend under the two control cases. However, compared to the no-control case, the coordinated control case presents $84.4 \%$ improvement in the average travel speeds of ramp 1.

In addition, the travel speed data of ramp 2 is presented in Figure 9. Under the coordinated control case, ramps 1 and 2 are controlled by a coordinated ramp metering strategy. Therefore, the coordinated control effect can also be present in the travel speeds of ramp 2. During the period from $3600 \mathrm{~s}$ to $8650 \mathrm{~s}$, there is a clear distinction between the travel speed curves, where the coordinated control case shows significant improvement compared to the no-control case. The travel

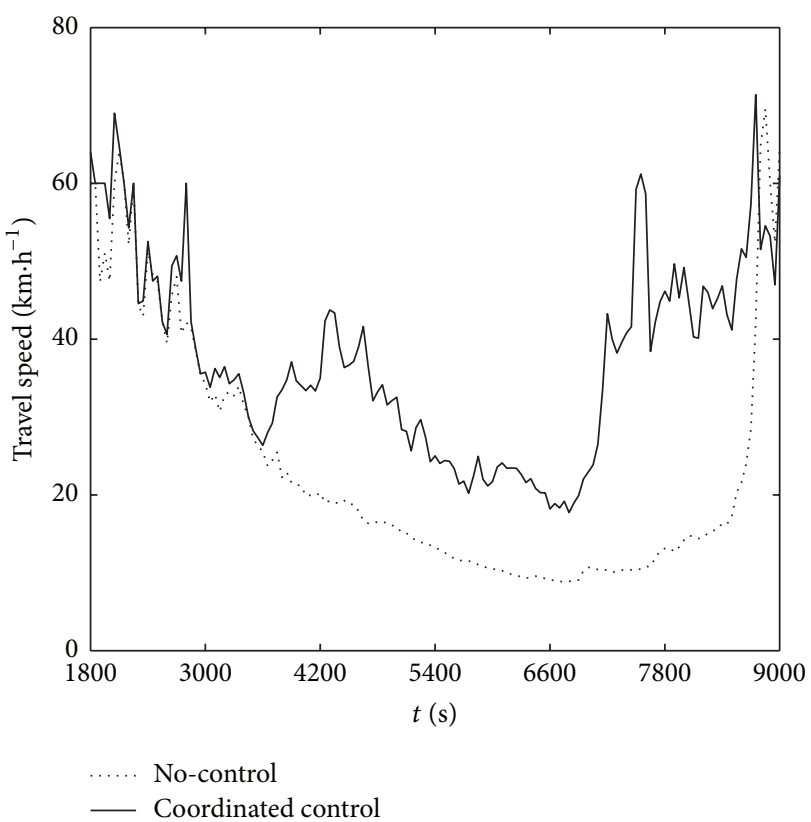

FIGURE 9: Ramp 2 travel speed.

speeds of ramp 2 during the peak period are about $33.2 \mathrm{~km} / \mathrm{h}$, under the coordinated control case, and about $18.86 \mathrm{~km} / \mathrm{h}$ higher than in the no-control case. The queue length variables in different segments of the studied network are shown in Figure 10.

Traffic congestion seems serious in the studied network during the peak period. Some different phenomena appear in the two cases, as the inflow of the studied network increases. Comparison and analysis are presented as follows.

4.2.1. When No-Control Method Is Applied in the Studied Network. In the no-control case, the overflow of the studied network causes serious traffic congestion in the merging region. The queuing situations in mainline $\mathrm{C}$ and on-ramp 3 are presented in Figures 10(a) and 10(b). Disturbance exists in the merging region due to vehicles on mainline $\mathrm{C}$ and on-ramp 3 competing for priority going into the merging region, which leads to a long queue in the studied network. Once the queue length becomes critical, the traffic flow of the transition region is affected. When the time is around $3000 \mathrm{~s}$, the queue length in on-ramp 3 exceeds the length of on-ramp 3 , and queuing vehicles reach the transition region, as shown in Figures 10(b) and 10(c).

At around $4400 \mathrm{~s}$, the transition region queue length reaches 980 meters, while the traffic problem at ramps 1 and 2 appears, meaning that both ramps 1 and 2 are not allowed to flow into the transition region, and then the queue occurs in both ramp 1 and ramp 2. During the period from $4400 \mathrm{~s}$ to $6800 \mathrm{~s}$, the maximum queue lengths of ramps 1 and 2 reach 568 meters and 397 meters, respectively. The average queue lengths of ramps 1 and 2 are 376 meters and 238 meters, respectively. At around $5000 \mathrm{~s}$, the queue reaches ramp 2 upstream, resulting in disturbances in traffic flow in the mainline $\mathrm{B}$. 

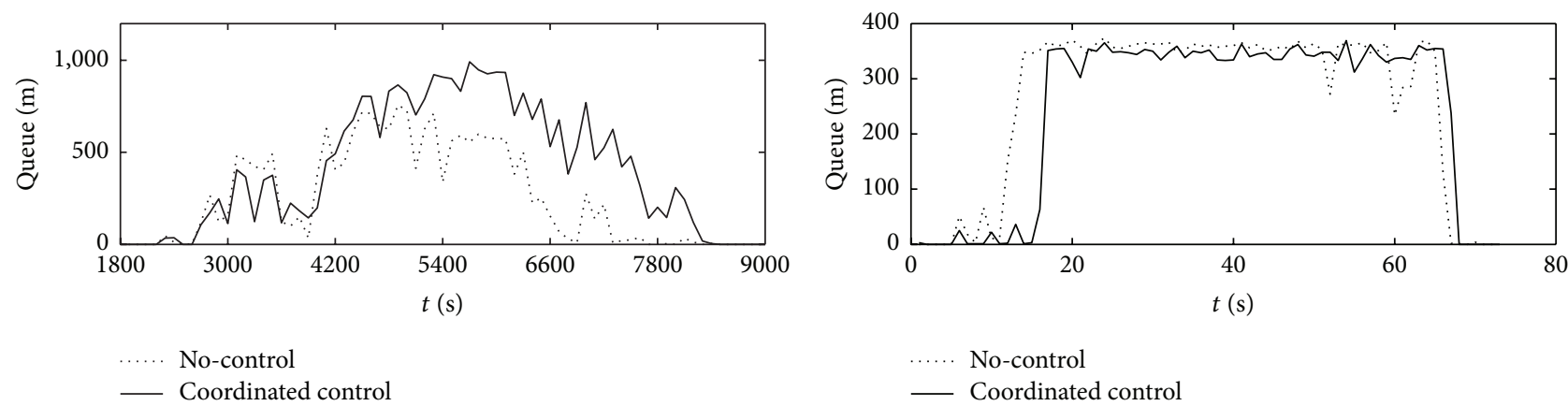

(a) Mainline C
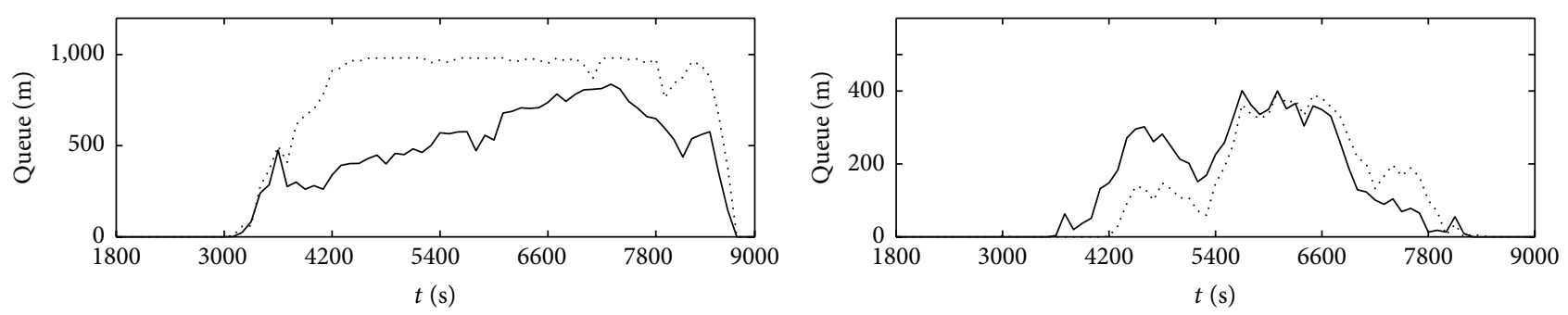

No-control

— Coordinated control

No-control

— Coordinated control

(c) Transition region

(d) Ramp 1

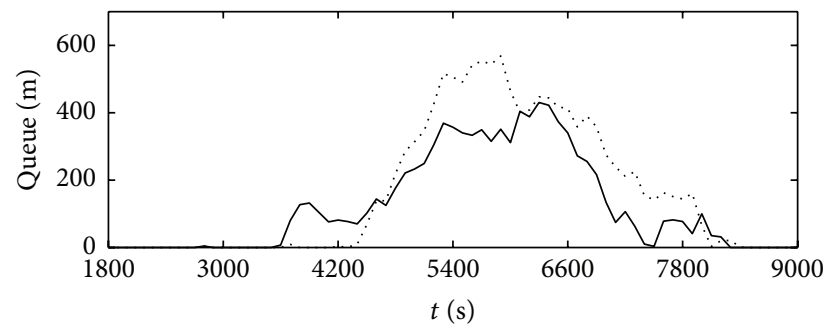

No-control

- Coordinated control

(e) Ramp 2

FIgURE 10: Queues in freeway network.

In the no-control case, traffic congestion in the studied network is serious. The total delay in the studied network is $1447950 \mathrm{veh} \cdot \mathrm{s}$, and the total delays in mainline C, ramp 1, and ramp 2 are $591733 \mathrm{veh} \cdot \mathrm{s}, 408169 \mathrm{veh} \cdot \mathrm{s}$, and $449210 \mathrm{veh} \cdot \mathrm{s}$, respectively.

4.2.2. When the Coordinated Control Method Proposed in This Paper Is Applied in the Studied Network. The total delay in the studied network is $1278540 \mathrm{veh} \cdot \mathrm{s}$, which is $11.7 \%$ improvement compared to the no-control case. Specifically, the total delays in ramps 1 and 2 show $18.0 \%$ and $25.1 \%$ improvements compared to the no-control case, respectively.

During the period from $1800 \mathrm{~s}$ to $2200 \mathrm{~s}$, the traffic operation situation is consistent with the no-control case. At around $2200 \mathrm{~s}$, disturbance in the merging region becomes serious, and the coordinated control is triggered. The VSL values and metering rates of the ramps are shown in Figures 11 and 12 , respectively.

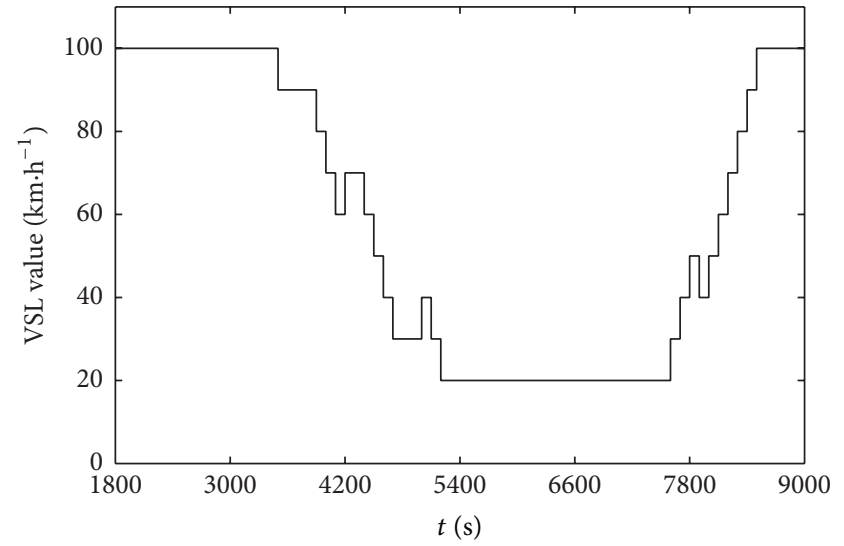

FIGURE 11: VSL values.

In the coordinated control case, the queue lengths in both mainline $\mathrm{C}$ and on-ramp 3 are lower than those in 

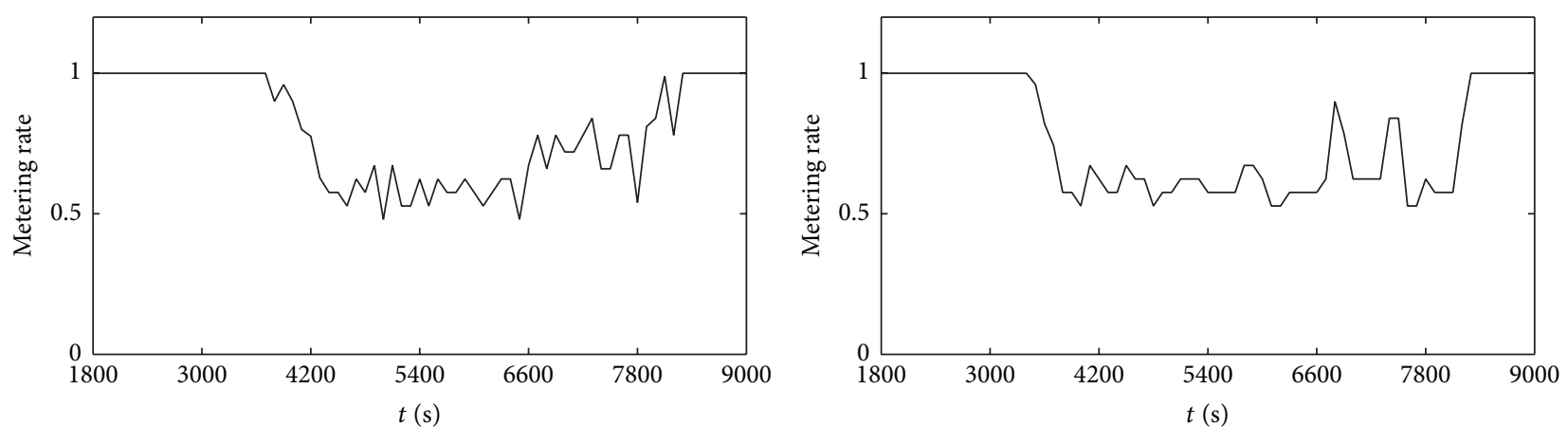

Ramp 1

- Ramp 2

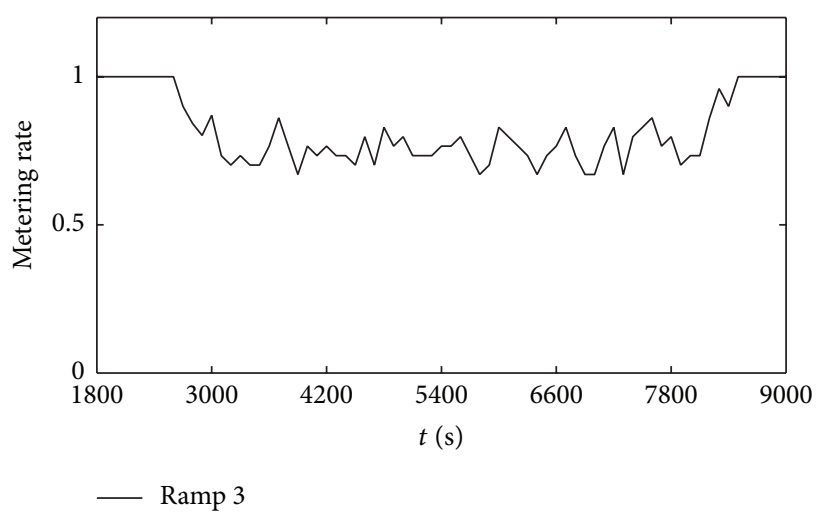

FIGURE 12: Metering rates at ramps.

the no-control case. At around $3100 \mathrm{~s}$, queuing vehicles arrive to the transition region. There is a time delay due to the process of the queue length forming in on-ramp 3 compared to the no-control case, because the effective control method and strategy are applied in the studied network. However, with the increase in the studied network inflow, the queue becomes longer. At around $3600 \mathrm{~s}$, the coordinated ramp metering in ramps 1 and 2 is activated, and congestion in the transition region is relieved. During the period from $3600 \mathrm{~s}$ to $7600 \mathrm{~s}$, the average queue length in ramps 1 and 2 is 209 meters and 270 meters, respectively. The maximum queue lengths are 401 meters and 430 meters, respectively.

In Figures 10(d) and 10(e), in the $4500 \mathrm{~s} \sim 6000 \mathrm{~s}$ time period, the queue in ramp 1 is longer than that under the nocontrol case, yet the queue length in ramp 2 is lower than that under the no-control case. The reason is that more right of way is given to ramp 2 vehicles flowing into the transition region under the coordinated control case.

\section{Conclusion}

A new coordinated control method is presented in this paper, which involves VSL control and coordinated ramp metering. An actual regional network in China is selected to verify rationality and validity of the proposed coordinated control method. The no-control and coordinated control cases are adopted in simulations. The results show that the coordinated control method presents better results in reducing the total delay in the regional network and enhancing the maximum merging region outflow. The coordinated control method leads to $11.7 \%$ improvement in the total delay compared to the no-control case.

In ongoing research we plan to consider different control methods including the coordinated method to further enhance the model. Different control time periods should also be considered in future research.

\section{Disclosure}

All statements of this paper are the sole responsibility of the authors and do not necessarily reflect the standard, specification, or regulation.

\section{Competing Interests}

The authors declare that they have no competing interests.

\section{Acknowledgments}

This work was partly funded by the Technological Innovation Program of the Shandong Province-administered Enterprises (20122150251-1).

\section{References}

[1] C. Roncoli, M. Papageorgiou, and I. Papamichail, "Traffic flow optimisation in presence of vehicle automation and communication systems-part II: optimal control for multi-lane motorways," Transportation Research Part C: Emerging Technologies, vol. 57, pp. 260-275, 2015. 
[2] L. D. Baskar, B. De Schutter, J. Hellendoorn, and Z. Papp, "Traffic control and intelligent vehicle highway systems: a survey," IET Intelligent Transport Systems, vol. 5, no. 1, pp. 3852, 2011.

[3] C. Pasquale, I. Papamichail, C. Roncoli, S. Sacone, S. Siri, and M. Papageorgiou, "Two-class freeway traffic regulation to reduce congestion and emissions via nonlinear optimal control," Transportation Research Part C: Emerging Technologies, vol. 55, no. 1, pp. 85-99, 2015.

[4] B. Ran and D. Boyce, Dynamic Urban Transportation Network Models: Theory and Implications for Intelligent Vehicle-Highway Systems, Springer Science \& Business Media, 2012.

[5] L. Zheng, S.-Q. Zhong, and S.-F. Ma, "Controlling traffic jams on a two-lane road using delayed-feedback signals," Journal of Zhejiang University: Science A, vol. 13, no. 8, pp. 620-632, 2012.

[6] P. Kachroo and K. M. A. Özbay, Feedback Ramp Metering in Intelligent Transportation Systems, Springer Science \& Business Media, 2011.

[7] M. Abdel-Aty, J. Dilmore, and A. Dhindsa, "Evaluation of variable speed limits for real-time freeway safety improvement," Accident Analysis and Prevention, vol. 38, no. 2, pp. 335-345, 2006.

[8] Y. Jo, H. Choi, S. Jeon, and I. Jung, "Variable speed limit to improve safety near traffic congestion on urban freeways," in Proceedings of the IEEE International Conference on Information Science and Technology (ICIST '12), pp. 43-50, Hubei, China, March 2012.

[9] G.-L. Chang, S. Y. Park, and J. Paracha, "Intelligent transportation system field demonstration: integration of variable speed limit control and travel time estimation for a recurrently congested highway," Transportation Research Record, no. 2243, pp. 55-66, 2011.

[10] R. Yu and M. Abdel-Aty, "An optimal variable speed limits system to ameliorate traffic safety risk," Transportation Research Part C: Emerging Technologies, vol. 46, pp. 235-246, 2014.

[11] H. Zhang, Z. Li, P. Liu, C. Xu, and H. Yu, "Control strategy of variable speed limits for improving traffic efficiency at merge bottleneck on freeway," Procedia-Social and Behavioral Sciences, vol. 96, pp. 2011-2023, 2013.

[12] F. Habtemichael and L. De Picado Santos, "Safety and operational benefits of variable speed limits under different traffic conditions and driver compliance levels," Transportation Research Record, no. 2386, pp. 7-15, 2013.

[13] M. Hadiuzzaman and T. Z. Qiu, "Cell transmission model based variable speed limit control for freeways," Canadian Journal of Civil Engineering, vol. 40, no. 1, pp. 46-56, 2013.

[14] M. Islam, M. Hadiuzzaman, J. Fang, T. Qiu, and K. El-Basyouny, "Assessing mobility and safety impacts of a variable speed limit control strategy," Transportation Research Record, vol. 2364, pp. $1-11,2013$.

[15] B. Kerner, "Study of freeway speed limit control based on threephase traffic theory," Transportation Research Record, vol. 1999, 2015.

[16] X.-Y. Lu, P. Varaiya, R. Horowitz, D. Su, and S. E. Shladover, "Novel freeway traffic control with variable speed limit and coordinated ramp metering," Transportation Research Record, no. 2229, pp. 55-65, 2011.

[17] R. C. Carlson, D. Manolis, I. Papamichail et al., "Integrated ramp metering and mainstream traffic flow control on freeways using variable speed limits," Procedia-Social and Behavioral Sciences, vol. 48, pp. 1578-1588, 2012.
[18] D. Li, P. Ranjitkar, and A. Ceder, "Integrated approach combining ramp metering and variable speed limits to improve motorway performance," in Proceedings of the 93rd Annual Meeting of Transportation Research Board, 2014.

[19] A. Hegyi, B. De Schutter, and H. Hellendoorn, "Model predictive control for optimal coordination of ramp metering and variable speed limits," Transportation Research Part C: Emerging Technologies, vol. 13, no. 3, pp. 185-209, 2005.

[20] R. C. Carlson, I. Papamichail, and M. Papageorgiou, "Integrated feedback ramp metering and mainstream traffic flow control on motorways using variable speed limits," Transportation Research Part C: Emerging Technologies, vol. 46, pp. 209-221, 2014.

[21] A. Nissan and H. N. Koutsopoulosb, "Evaluation of the impact of advisory variable speed limits on motorway capacity and level of service," Procedia-Social and Behavioral Sciences, vol. 16, pp. 100-109, 2011.

[22] D. Su, X. Lu Y, P. Varaiya et al., "Variable speed limit and ramp metering design for congestion caused by weaving," in Proceedings of the 90th Annual Meeting of the Transportation Research Board (TRB '11), Washington, DC, USA, January 2011.

[23] M. Papageorgiou and M. Marinaki, A Feasible Direction Algorithm for the Numerical Solution of Optimal Control Problems, Dynamic Systems \& Simulation Laboratory, Technical University of Crete, Chania, Greece, 1995.

[24] I. Schelling, A. Hegyi, and S. P. Hoogendoorn, "SPECIALIST$\mathrm{RM}$ - integrated variable speed limit control and ramp metering based on shock wave theory," in Proceedings of the 14th IEEE International Intelligent Transportation Systems Conference (ITSC '11), pp. 2154-2159, IEEE, Washington, Wash, USA, October 2011.

[25] Y. Li and A. H. F. Chow, "Optimisation of motorway operations via ramp metering and variable speed limits," Transportation Planning and Technology, vol. 38, no. 1, pp. 94-110, 2015.

[26] M.-H. Ma, Q.-F. Yang, S.-D. Liang, and Z.-L. Li, "Integrated variable speed limits control and ramp metering for bottleneck regions on freeway," Mathematical Problems in Engineering, vol. 2015, Article ID 313089, 17 pages, 2015.

[27] D. Li, Towards an Efficient and Equitable Motorway System Using Ramp Metering and Variable Speed Limits, 2015.

[28] S. Zhao, S. Liang, H. Liu, and M. Ma, "CTM based real-time queue length estimation at signalized intersection," Mathematical Problems in Engineering, vol. 2015, Article ID 328712, 12 pages, 2015. 


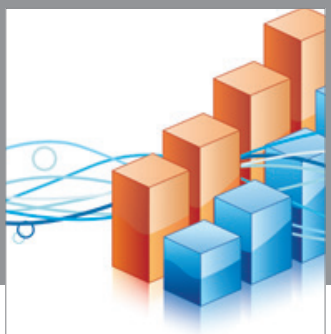

Advances in

Operations Research

vatem alat4

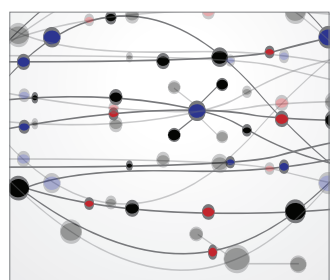

\section{The Scientific} World Journal
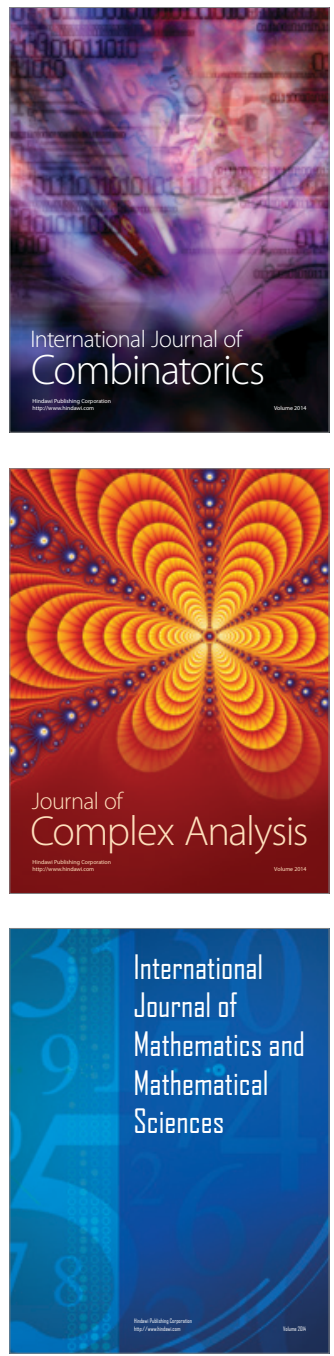
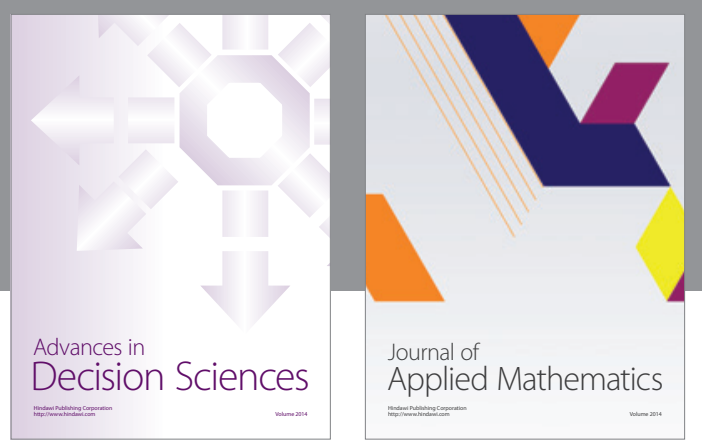

Algebra

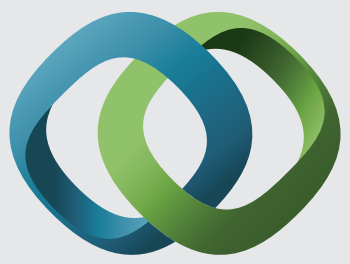

\section{Hindawi}

Submit your manuscripts at

http://www.hindawi.com
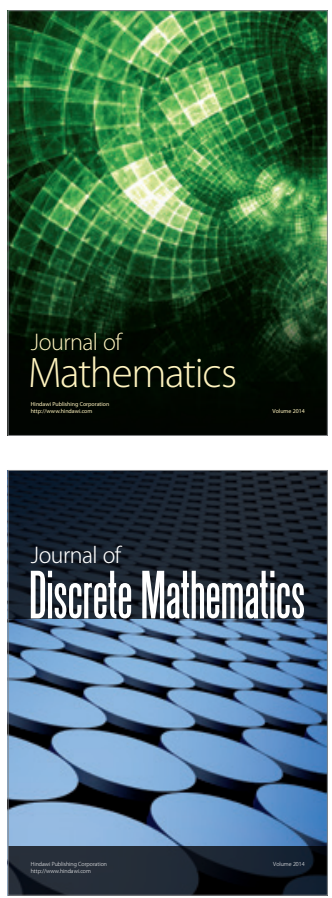

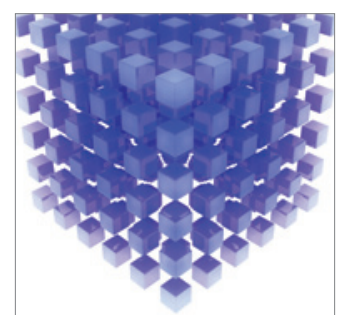

Mathematical Problems in Engineering
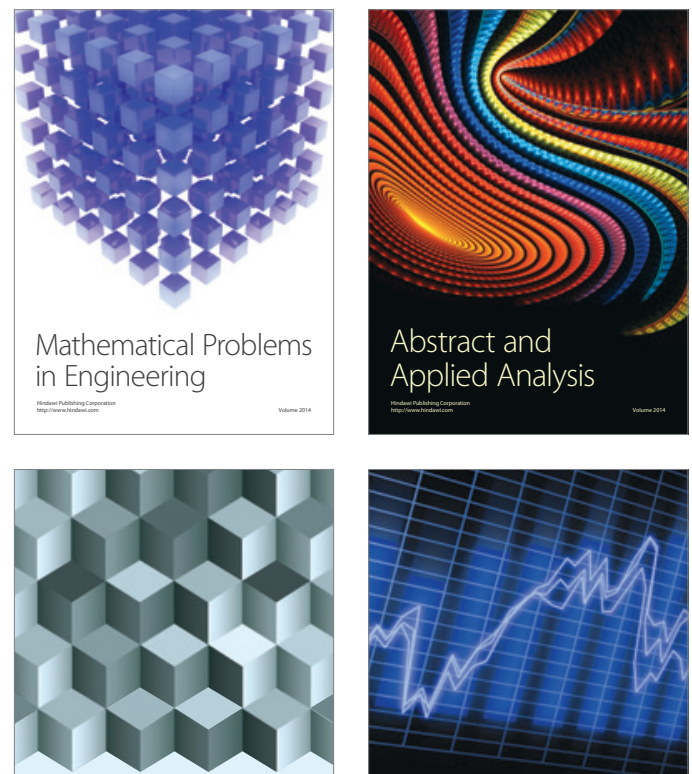

Journal of

Function Spaces

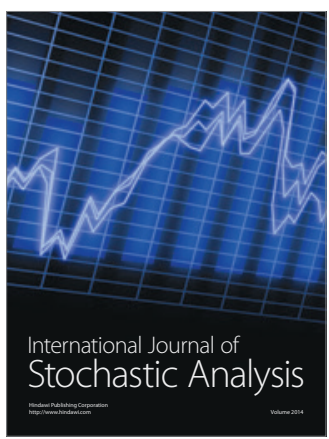

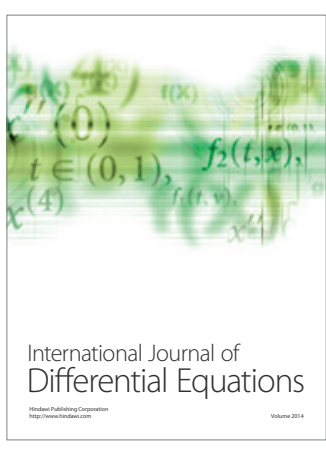
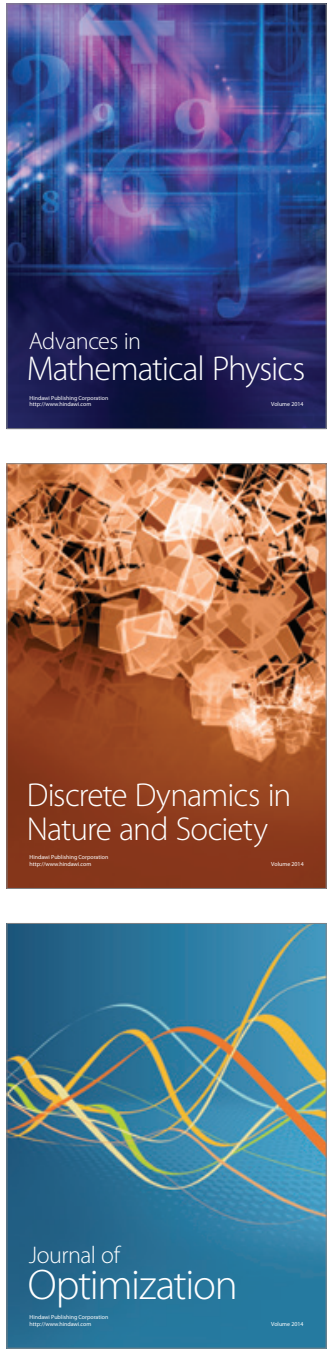